\title{
Diseño y análisis comparativo de un inventario de indicadores de mortalidad evitable adaptado a las condiciones sanitarias de Colombia
}

\author{
Rubén Darío Gómez-Arias, ${ }^{1}$ Andreu Nolasco Bonmatí, ${ }^{2}$ \\ Pamela Pereyra-Zamora, ${ }^{2}$ Samuel Arias-Valencia, ${ }^{1,3}$ \\ Fabio León Rodríguez-Ospina ${ }^{1}$ y Daniel Camilo Aguirre ${ }^{1}$
}

Forma de citar Gómez-Arias RD, Nolasco Bonmatí A, Pereyra-Zamora P, Arias-Valencia S, Rodríguez-Ospina FL, Aguirre DC. Diseño y análisis comparativo de un inventario de indicadores de mortalidad evitable adaptado a las condiciones sanitarias de Colombia. Rev Panam Salud Publica. 2009;26(5):385-97.

RESUMEN Objetivos. Elaborar un inventario de indicadores de mortalidad evitable (INIME) que permita analizar las fallas en el control de los riesgos de mortalidad predominantes en Colombia y comparar los resultados de su aplicación con los obtenidos mediante dos enfoques ampliamente utilizados.

Métodos. Se revisaron los registros oficiales de mortalidad de Colombia de 1985 a 2001; las causas básicas de muerte se clasificaron según la CIE-9. Se seleccionaron los indicadores de mortalidad evitable (ME) mediante un algoritmo que combinó las listas de Holland y de Taucher, la definición de Rutstein y colaboradores y el principio de Uemura. Se compararon las proporciones de muertes evitables resultantes de aplicar el INIME y las dos listas de ME a una base de datos con los registros oficiales de defunciones de Colombia de 1993 a 1996.

Resultados. De las 680617 defunciones registradas en el período de estudio, se clasificaron como evitables 18,2\% según la lista de Holland y 51,3\% según la lista de Taucher. La ME según el INIME ascendió a 76,7\%. Este patrón se mantuvo relativamente estable entre 1993 y 1996. Las diferencias observadas en la proporción de muertes evitables según el INIME y las dos listas de $M E$ se relacionaron con el perfil epidemiológico local y el enfoque conceptual de cada lista. Conclusiones. Las diferencias entre el INIME y las listas de ME de Holland y de Taucher muestran las consecuencias de usar una u otra clasificación en el contexto colombiano. El INIME puede constituir un recurso valioso para fundamentar y evaluar políticas sanitarias, pero debe ajustarse a la situación específica en que se aplique.

Palabras clave Mortalidad; evaluación en salud; políticas públicas en salud; inequidad social; Colombia.

1 Universidad de Antioquia, Facultad Nacional de Salud Pública, Antioquia, Colombia. La correspondencia se debe dirigir a Rubén Darío Gómez-Arias, Universidad de Antioquia, Facultad Nacional de Salud Pública, Calle 62 No 52-29, Medellín, Colombia. Correos electrónicos: rubengomez33@gmail. com,rdgomez@guajiros.udea.edu.co

2 Universidad de Alicante, Unidad de Investigación de Análisis de la Mortalidad y Estadísticas Sanitarias, Alicante, Valencia, España.

3 Instituto Nacional de Cancerología, Santa Fe de Bogotá, Colombia.
El informe Salud Mundial 2000 de la Organización Mundial de la Salud (OMS) generó un intenso debate sobre las políticas públicas que afectan a la salud y los criterios para su evaluación (1). Desde mediados de 1980 ha ganado terreno la idea de utilizar pruebas para evaluar las políticas públicas (2-5) y, en este campo, el análisis de la mortalidad evitable (ME) constituye uno de los recursos disponibles más importantes, tanto por la trascendencia de la muerte para la sociedad como por la relativa disponibilidad y solidez de las fuentes de datos (6).

Aunque la muerte es un hecho inevitable, varias de las causas que llevan a ella han logrado controlarse $\mathrm{y}$, en ese 
sentido, las defunciones por estas causas se consideran muertes evitables. Desde 1970 se ha avanzado notablemente en el estudio de la ME y en la literatura científica se reconoce su importancia para evaluar las políticas (7-9). Sin embargo, los criterios y los procedimientos de análisis utilizados aún presentan limitaciones, ya que las clasificaciones de la ME suelen basarse en la opinión de expertos, no existe una prueba de oro para clasificarlas y los criterios de ME utilizados con éxito en un contexto pueden ser poco relevantes en otras poblaciones y en otros períodos $(6,10-12)$. Tampoco existe un modelo aceptado como estándar para establecer el carácter evitable de una muerte y las publicaciones rara vez fundamentan explícitamente la razón para incluir o no una causa de muerte como evitable. Hasta la fecha no hay un estudio publicado que haya comparado sistemáticamente los criterios e indicadores propuestos desde los diferentes enfoques $(6,13)$.

Los múltiples enfoques, mediciones y usos de la ME documentados (6, 10-12) pueden agruparse en tres categorías: a) análisis de la ME a partir de listas de causas evitables definidas por grupos de expertos; este enfoque predomina en la literatura y suele centrarse en las defunciones cuya prevención y control se atribuyen a la atención médica (AM); b) análisis de la ME a partir de la efectividad de la tecnología disponible; supone que no todas las muertes evitables lo son en el mismo grado y que es indispensable ajustar los resultados según la efectividad ${ }^{4}$ relativa de las intervenciones; y c) análisis de la ME a partir del exceso de mortalidad en una población dada con respecto a una población estándar con la menor tasa de mortalidad observada.

La mayoría de las definiciones de ME suelen basarse en el criterio de Rutstein y colaboradores: "una muerte se considera evitable cuando, si todo lo que se tenía que hacer se hubiera hecho, tal defunción se hubiera prevenido o retrasado" (14). Este criterio acepta de forma implícita la necesidad de que exista por lo menos una intervención capaz de modificar los factores determinantes de la muerte. Posteriormente, Holland propuso aceptar el carácter evitable de una muerte cuando puedan identificarse no

\footnotetext{
4 En este artículo se utiliza el término "efectividad" para calificar el efecto de intervenciones realizadas en condiciones no controladas.
}

solo una intervención efectiva, sino también algún proveedor de la misma (15) que, desde su perspectiva, debía ser un proveedor de AM. Así, el Atlas de Mortalidad Evitable de la Comunidad Europea, elaborado bajo la dirección de Holland (16), definió dos categorías de causas evitables: las asociadas con la $\mathrm{AM}$, es decir, las que se evitarían por acciones de prevención secundaria o tratamiento médico, y las asociadas con elementos de la política nacional de salud, que se evitarían por acciones de prevención primaria. En las diferentes versiones de este atlas se parte de que las causas evitables lo son solo en ciertos límites de edad, que la lista de ME no abarca todas las causas evitables y que las causas seleccionadas no son igualmente evitables en todas las personas (6). Las listas de ME publicadas por la literatura suelen basarse en los criterios de Rutstein y colaboradores (14) y de Holland (6).

Los enfoques sobre la ME basados en la efectividad de las intervenciones suponen que una misma causa de muerte puede obedecer de forma distinta a acciones similares de control y que los indicadores de la ME deben ajustarse según su grado de respuesta a las intervenciones disponibles. Tal como lo reconocen sus proponentes, la dificultad de este enfoque radica en la falta de investigaciones que den cuenta de la efectividad de las intervenciones para reducir la mortalidad $(6,10,11,15)$. Los enfoques que proponen analizar la ME desde el exceso de la mortalidad —más que desde una lista definida de causas - consideran, como Uemura (17), que las tasas mínimas de mortalidad logradas por una población reflejan su capacidad para evitar la muerte hasta ese punto y que si los recursos estuvieran equitativamente distribuidos todas las poblaciones deberían tener tasas similares. En consecuencia, las muertes que una sociedad ha logrado evitar debieran considerarse también evitables en las demás.

Las mediciones de la ME publicadas presentan variaciones geográficas y temporales que se han relacionado, por una parte, con los criterios utilizados para definir y calificar el carácter evitable de las defunciones (18-21) y, por otra, con los contextos epidemiológicos. Por esa razón, se recomienda establecer los indicadores de ME en función de las prioridades regionales y nacionales (22). En un sentido amplio, la mayoría de las defunciones podrían ser evitables en algún grado en la medida en que su cadena causal pueda interrumpirse en puntos controlables del proceso. Sin embargo, y aceptando los criterios de Holland, varios autores consideran que las listas de ME deben servir a una finalidad específica de tipo práctico $(6,10,11,15)$. En Europa, la ME ha sido una importante línea de estudio y la clasificación presentada por Holland es ampliamente utilizada desde 1986 como indicador de fallas en la AM, más que como reflejo de otros determinantes sociales (9). En ese sentido, las listas de ME apoyadas en los criterios de Holland suelen abarcar solo algunas de las causas evitables por AM predominantes en cada región o país (6).

El interés por la ME también ha crecido en América Latina, donde los estudios se han fundamentado, desde 1978, en la clasificación de Taucher $(23,24)$. Este enfoque trasciende el interés por detectar fallas en el sistema de AM y considera que ciertas causas de muerte pueden considerarse evitables no solo por la AM, sino también por el control de los riesgos ambientales y sociales. Aunque Taucher reconoce la dificultad de establecer la efectividad específica atribuible a cada una de las diferentes intervenciones, considera necesario proceder con criterios pragmáticos y agrupa las muertes evitables en cuatro categorías: a) evitables principalmente por acciones de prevención primaria; b) evitables principalmente por la AM; c) evitables principalmente por intervenciones sobre el ambiente; y d) evitables por la combinación de acciones del sistema de salud y de otros sectores.

Las listas de Holland $(9,10)$ y de Taucher $(12,23,24)$ coinciden en que las muertes consideradas evitables indican fallas en el control de los factores determinantes de la muerte, sin embargo, difieren profundamente en su enfoque con respecto a la ME y los indicadores seleccionados, lo que necesariamente conduce a diferencias en la proporción de la ME obtenida a partir de cada uno de esos enfoques e indicadores. Sus criterios, ampliamente utilizados en diferentes países, han mostrado su utilidad y sus limitaciones en cada región o país; sin embargo, hasta la fecha no se han hecho comparaciones que permitan establecer las consecuencias de aplicar un método u otro para evaluar políticas públicas.

La medición de la ME enfrenta dificultades adicionales por la variabilidad regional o local en el perfil epidemiológico 
y las características de la información disponible. Idealmente, los indicadores deben ser válidos, confiables, sensibles, específicos y útiles para orientar las decisiones (25) y por ello se deben definir operativamente de forma clara y precisa para que se puedan medir y controlar. No obstante, los problemas prácticos suelen estar lejos de las condiciones ideales y los datos rara vez están disponibles en la forma en que los tomadores de decisiones los requieren (26).

El objetivo del presente trabajo fue elaborar un inventario de indicadores de mortalidad evitable (INIME) que los tomadores de decisiones puedan utilizar, aisladamente o en su conjunto, para analizar las fallas en el control de los riesgos de mortalidad predominantes en Colombia y en otros países con condiciones epidemiológicas y sanitarias similares. Además, se analizan las diferencias entre el nuevo inventario y las listas de Holland y de Taucher y se comparan los resultados al aplicar los tres enfoques de ME en una misma población.

\section{MATERIALES Y MÉTODOS}

Para elaborar el INIME ajustado al contexto colombiano se revisaron los registros oficiales de mortalidad de Colombia de 1985 a 2001. Las causas básicas de muerte se agruparon según la Clasificación Estadística Internacional de Enfermedades y Problemas Relacionados con la Salud, revisión 9 (CIE-9) (27). Las causas de muerte más frecuentes durante el período fueron: homicidios $(15,5 \%)$, enfermedad isquémica del corazón $(12,3 \%)$, tumores $(14,0 \%)$-fundamentalmente de estómago $(2,3 \%)$, vías respiratorias $(1,4 \%)$, próstata $(0,8 \%)$ y cuello uterino $(0,8 \%)$ - y enfermedades infecciosas y parasitarias $(3,9 \%)$. En general, $42,5 \%$ de las defunciones registradas correspondía a mayores de 65 años.

Una vez establecido el perfil de la mortalidad, se procedió a definir un sistema de clasificación de la ME que pudiera aplicarse a las causas de muerte predominantes. Para ello, en lugar de recurrir a un grupo de expertos para cons- truir el INIME, se seleccionaron los indicadores de las listas de ME según Holland $(9,10)$ y Taucher $(12,23,24)$, ya que ambas han logrado un buen consenso internacional. La comparación de ambas listas por grupos de causas mostró que en cada una de ellas se contemplan criterios diferentes para definir el carácter evitable de las muertes más frecuentes y prioritarias de atención en Colombia. En consecuencia, para responder más cabalmente a las condiciones epidemiológicas locales en este estudio se decidió integrar los dos enfoques mediante un algoritmo (28) que combinó las listas de Holland y de Taucher, la definición de Rutstein y colaboradores (14) y el principio de Uemura $(17,29)$ (figura 1). Así, cada causa de mortalidad registrada durante el período se clasificó como evitable o no evitable según ese algoritmo.

En una primera etapa, todos los códigos de la CIE-9 se clasificaron según la lista de causas de muerte de Holland como evitables (CMEH) o no evitables (CMNEH); el procedimiento se realizó de manera auto-

\section{FIGURA 1. Algoritmo de clasificación de las defunciones según su condición de evitable}

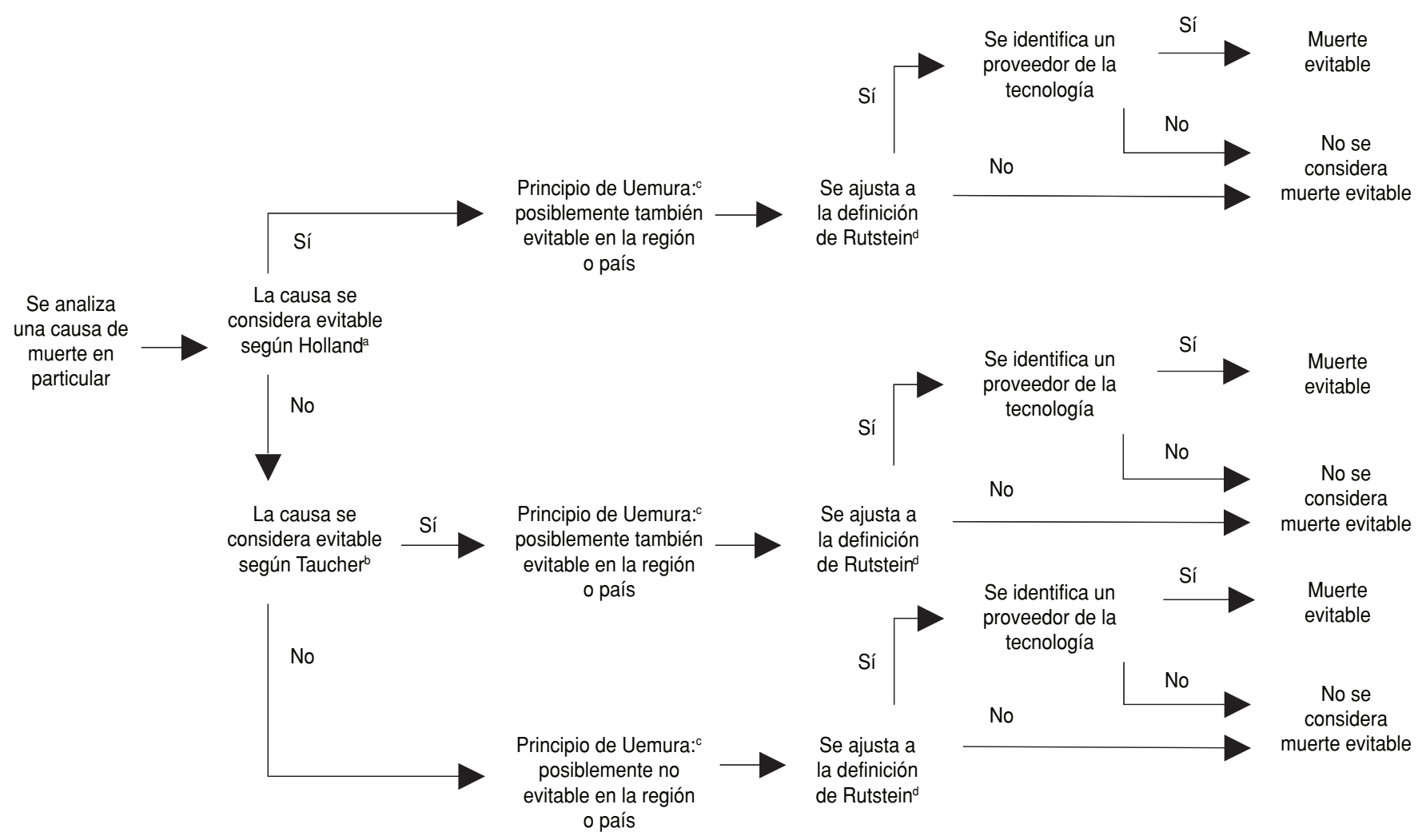

Fuente: Ref. 28.

a Véanse las referencias 9 y 10.

b Véanse las referencias 12, 23 y 24

c Véanse las referencias 17 y 29.

d Véase la referencia 14. 
matizada con el programa informático SPSS versión 10 por reclasificación de variables. A partir del principio de Uemura, toda CMEH se consideró potencialmente evitable también para Colombia. Se verificó que cada CMEH se ajustara a la definición de Rutstein y colaboradores y que existiera en el país algún proveedor de tecnología potencialmente efectiva que se pudiera aplicar, si bien dicho proveedor podía actuar en cualquiera de los sectores productores de bienes o servicios, estatales o privados, y no solamente en el sector salud. Las causas clasificadas en la primera fase como $\mathrm{CMEH}$ se incorporaron directamente al INIME, mientras que los códigos clasificados como CMNEH en esta primera etapa se clasificaron según la lista de Taucher en causas de muerte evitables (CMET) y no evitables (CMNET). Las CMNEH que resultaron CMET se agregaron al INIME siempre y cuando se identificara en el país algún proveedor de tecnologías aplicables. Para clasificar una causa como evitable no se tuvo en cuenta la edad. Si no se llegaba a un criterio que justificara la inclusión de una causa de muerte en el inventario de indicadores de $\mathrm{ME}$, esta se consideró no evitable.

Se identificó al menos un proveedor de tecnología activo en Colombia para cada código clasificado como $\mathrm{CMEH}$ o CMET. Las CMNEH y CMNET que se ajustaban al criterio de Rutstein y colaboradores y para las que se identificó alguna tecnología de control potencialmente efectiva con un proveedor dentro del país se incorporaron al inventario de indicadores de ME (cuadro 1). Como resultado de estos procedimientos se obtuvo un INIME compuesto por todas las $\mathrm{CMEH}$, todas las CMET $\mathrm{y}$, adicionalmente, todas las CMNEH y CMNET que se ajustaban al criterio de Rutstein y colaboradores y podían considerarse evitables en Colombia.

Una vez elaborado el INIME, se compararon las proporciones de muertes evitables resultantes de aplicar esos criterios y las listas de ME de Holland y de Taucher a una base de datos con todos los registros oficiales de defunciones de Colombia de 1993 a 1996, clasificados a tres dígitos según la CIE-9. Se seleccionó este período por la vigencia de las listas a contrastar y porque no hubo cambios en los sistemas de clasificación o registro de los fallecimientos. La clasificación de las defunciones registradas según el INIME y las dos listas se realizó automatizada e independientemente por reclasificación de variables, según los códigos de la CIE-9. Las muertes evitables resultantes según cada clasificación se compararon por sexo, edad y causa básica de muerte. Al final, las causas evitables se agruparon según las cuatro categorías de ME de Taucher, por considerar que este modelo se ajustaba mejor, tanto a un enfoque multicausal de la muerte como a una visión más integral de las políticas públicas.

\section{RESULTADOS}

A partir de las listas originales de Holland $y$ Taucher $y$ la lista adicional de causas de ME agregadas (cuadro 1) se obtuvo el INIME según los códigos de la CIE-9 y la CIE-10 (cuadro 2).

De las 680617 defunciones registradas en el período de estudio, $18,2 \%$ se clasificaron como $\mathrm{CMEH}$ (cuadro 3), mientras que de los 556793 registros restantes, $49,7 \%$ se clasificaron como CMET. Del total de registros, solamente $10,6 \%$ era simultáneamente CMEH y CMET, 40,7\% era CMET y 7,6\% era CMEH; $41,1 \%$ de las muertes registradas se consideraron no evitables según ambos criterios.

La comparación de los resultados evidenció las diferencias conceptuales, ventajas y limitaciones de las listas de Holland y de Taucher sobre el carácter evitable de la mortalidad en la población estudiada. A diferencia de las $\mathrm{CMEH}$, en las que predominan las causas de muerte relacionadas en mayor medida con la $\mathrm{AM}$, en la lista de Taucher se tienen en cuenta otros determinantes de la muerte, de manera que $51,3 \%$ de las defunciones analizadas se clasificó como ME - la mayoría de ellas homicidios y otras muertes evitables mediante medidas mixtas- y solo se hubiera podido prevenir 14,3\% mediante acciones de AM.

La magnitud de las discrepancias entre las listas de Holland y de Taucher varió con la edad, el sexo y la causa de muerte (cuadro 3). El acuerdo entre los resultados obtenidos con el INIME y ambas listas fue bajo en la mayoría de los casos y las diferencias fueron mayores en los grupos de causas de muerte predominantes en la población analizada. La diferencia más importante entre ambas clasificaciones se observó en las muertes violentas, especialmente en hombres por ser el grupo más afectado por este problema. Otra diferencia importante se vinculó con la edad, ya que, a diferencia de la lista de Holland, en la lista de Taucher se considera que las muertes en ma- yores de 64 años pueden ser evitables si la causa básica lo es, y consecuentemente $34,1 \%$ de las defunciones registradas en Colombia en este grupo de edad se clasificó como ME. Finalmente, en la lista de Taucher se clasifica como ME una importante proporción de defunciones colombianas no incluidas en el Atlas Europeo, como la desnutrición, la tuberculosis, la diarrea, la malaria y los trastornos perinatales, además de $34,1 \%$ de las defunciones en personas de 65 años o más. Por otra parte, según la lista de Holland, Colombia presenta una proporción importante de muertes por afecciones cardiovasculares reconocidas en Europa como causas de ME y que escapan a la lista de Taucher. Al elaborar el INIME se tuvo en cuenta que no considerar alguna de las causas ampliamente reconocidas como evitables en otras regiones puede enmascarar condiciones de inequidad de especial importancia al evaluar políticas públicas.

La ME según el INIME ascendió a $76,7 \%$ de todas las defunciones registradas en el período de estudio (cuadro 3). Este valor es mayor que los obtenidos según las listas de Taucher y de Holland en $25,4 \%$ y $58,5 \%$, respectivamente. Este patrón se mantuvo relativamente estable durante los cuatro años analizados y en la mayoría de los grupos de edad, especialmente en los grupos de causas más frecuentes en la población y en los varones. La proporción de muertes evitables según el INIME fue mayor que la obtenida según las $\mathrm{CMEH}$, especialmente en los varones de 15 a 44 años -debido principalmente a los homicidios y muertes por causas externas- y entre los mayores de 65 años, ya que es la edad límite establecida por Holland para reconocer la condición de evitable de las muertes.

Aunque el objetivo de este estudio no era analizar la ME en Colombia y solo se estudiaron cuatro años, se exploró el comportamiento de la $\mathrm{ME}$ con respecto a la mortalidad por causas consideradas no evitables. Según el INIME, las muertes evitables disminuyeron en Colombia durante el período analizado, con un porcentaje de cambio medio anual (PCA) de $-1,4 \%$, mientras las no evitables se redujeron menos $(\mathrm{PCA}=-0,9 \%)$; la serie estudiada es muy corta para establecer intervalos de confianza. También se redujeron los valores de las muertes evitables por medidas de prevención primaria (PCA $=-8,9 \%$ ) $\mathrm{y}$ por $\mathrm{AM}(\mathrm{PCA}=-3,9 \%)$; en cambio, 
CUADRO 1. Causas de muerte consideradas no evitables en las listas de Holland y de Taucher, incorporadas al inventario de indicadores de mortalidad evitable (INIME)

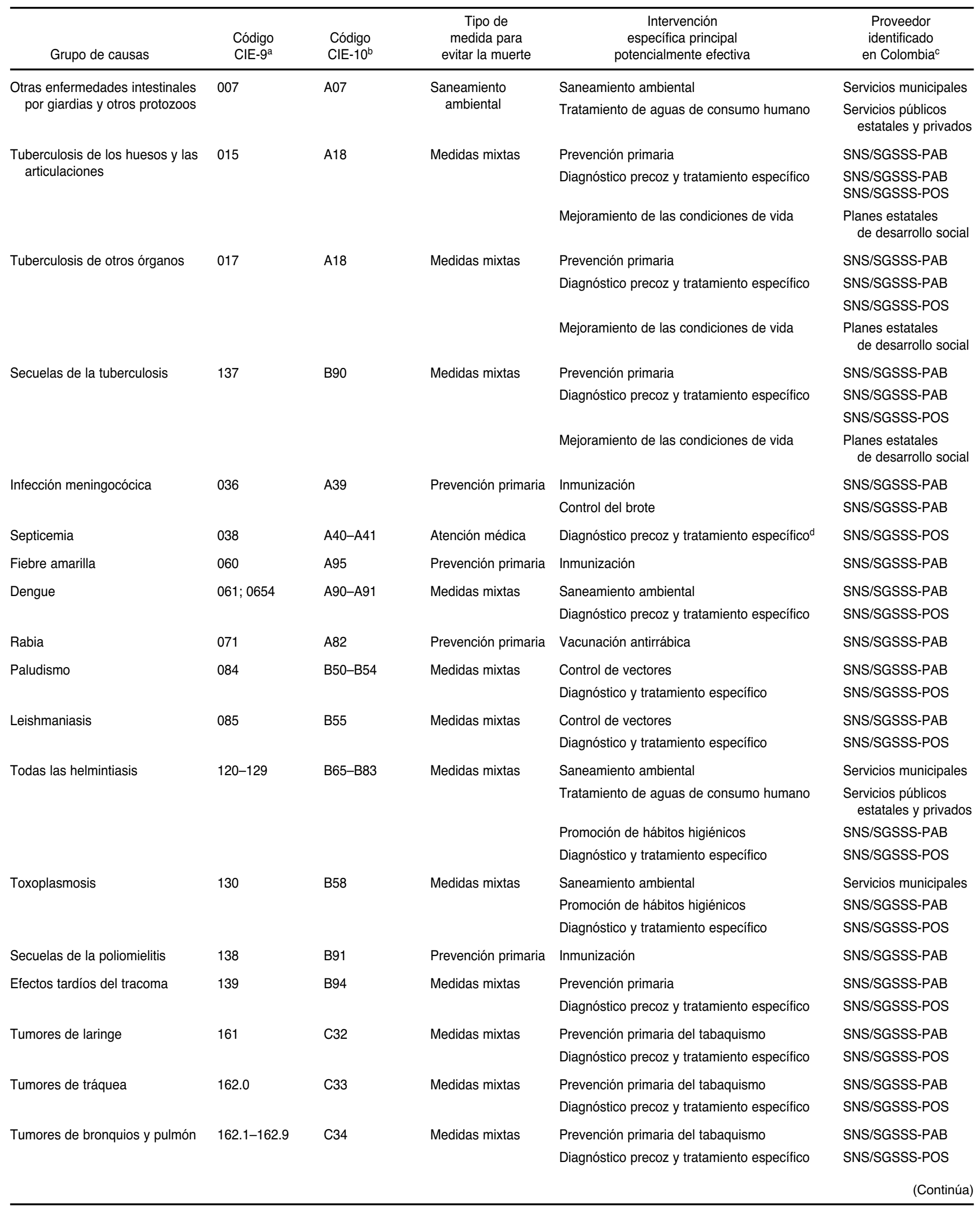


CUADRO 1. (Continuación)

\begin{tabular}{|c|c|c|c|c|c|}
\hline Grupo de causas & $\begin{array}{l}\text { Código } \\
\text { CIE-9a }\end{array}$ & $\begin{array}{l}\text { Código } \\
\text { CIE-10 }\end{array}$ & $\begin{array}{c}\text { Tipo de } \\
\text { medida para } \\
\text { evitar la muerte }\end{array}$ & $\begin{array}{c}\text { Intervención } \\
\text { específica principal } \\
\text { potencialmente efectiva }\end{array}$ & $\begin{array}{l}\text { Proveedor } \\
\text { identificado } \\
\text { en Colombia }\end{array}$ \\
\hline Hepatitis B & & $\begin{array}{l}\text { B160, B161, } \\
\text { B180, B181 }\end{array}$ & Prevención primaria & $\begin{array}{l}\text { Inmunización } \\
\text { Diagnóstico y tratamiento específico }\end{array}$ & $\begin{array}{l}\text { SNS/SGSSS-PAB } \\
\text { SNS/SGSSS-POS }\end{array}$ \\
\hline VIH-sida & $\begin{array}{l}279.5 ; 279.6 \\
\quad 795.8\end{array}$ & B20-B24 & Medidas mixtas & $\begin{array}{l}\text { Prevención primaria (desde 1984) } \\
\text { Diagnóstico precoz y tratamiento específico } \\
\text { (desde 1989) }\end{array}$ & $\begin{array}{l}\text { SNS/SGSSS-PAB } \\
\text { SNS/SGSSS-POS }\end{array}$ \\
\hline Secuelas de la lepra & & B92 & Medidas mixtas & Prevención primaria & SNS/SGSSS-PAB \\
\hline Delírium trémens & 291 & $d$ & Medidas mixtas & Control del consumo de psicoactivos & $\begin{array}{l}\text { SNS/SGSSS-PAB } \\
\text { SNS/SGSSS-POS }\end{array}$ \\
\hline $\begin{array}{l}\text { Síndrome de abstinencia de } \\
\text { la droga }\end{array}$ & 292 & $d$ & Medidas mixtas & Diagnóstico precoz y tratamiento específico & $\begin{array}{l}\text { SNS/SGSSS-PAB } \\
\text { SNS/SGSSS-POS }\end{array}$ \\
\hline Anemia por deficiencia de hierro & 280 & D50 & Medidas mixtas & $\begin{array}{l}\text { Mejoramiento de las condiciones de vida } \\
\text { Seguridad alimentaria (complementación con } \\
\quad \text { sulfato ferroso) } \\
\text { Diagnóstico precoz y tratamiento específico }\end{array}$ & $\begin{array}{l}\text { Planes estatales } \\
\text { de desarrollo social } \\
\text { SNS/SGSSS-PAB } \\
\text { SNS/SGSSS-POS }\end{array}$ \\
\hline $\begin{array}{l}\text { Cardiopatías reumáticas agudas } \\
\text { o crónicas }\end{array}$ & $391 ; 394-397$ & $101 ; 105-108$ & Medidas mixtas & Mejoramiento de las condiciones de vida & $\begin{array}{l}\text { Planes estatales } \\
\text { de desarrollo social }\end{array}$ \\
\hline & & & & $\begin{array}{l}\text { Seguridad alimentaria (complementación con } \\
\text { sulfato ferroso) }\end{array}$ & SNS/SGSSS-PAB \\
\hline & & & & Diagnóstico precoz y tratamiento específico & SNS/SGSSS-POS \\
\hline $\begin{array}{l}\text { Enfermedad hipertensiva y } \\
\text { cerebrovascular }\end{array}$ & $430-438$ & $160-169$ & Atención médica & Diagnóstico precoz y tratamiento específico & SNS/SGSSS-POS \\
\hline $\begin{array}{l}\text { Enfermedad isquémica del } \\
\text { corazón }\end{array}$ & $410-414$ & $120-125$ & Atención médica & Diagnóstico precoz y tratamiento específico & SNS/SGSSS-POS \\
\hline Valvulopatías & 424 & 134 & Atención médica & Diagnóstico precoz y tratamiento específico & SNS/SGSSS-POS \\
\hline Insuficiencia cardíaca congestiva & 428 & 150 & Atención médica & Diagnóstico precoz y tratamiento específico & SNS/SGSSS-POS \\
\hline Tromboflebitis & 453 & 182 & Atención médica & Diagnóstico precoz y tratamiento específico & SNS/SGSSS-POS \\
\hline Litiasis renal y ureteral & $592 ; 594$ & $\mathrm{~N} 20 ; \mathrm{N} 21$ & Atención médica & Diagnóstico precoz y tratamiento específico & SNS/SGSSS-POS \\
\hline Cistitis & 595 & N30 & Atención médica & Diagnóstico precoz y tratamiento específico & SNS/SGSSS-POS \\
\hline $\begin{array}{l}\text { Enfermedades de los órganos } \\
\text { genitales }\end{array}$ & $600-608$ & N40-N51 & Atención médica & Diagnóstico precoz y tratamiento específico & SNS/SGSSS-POS \\
\hline Trastornos de la mama & $610-611$ & N60-N64 & Atención médica & Diagnóstico precoz y tratamiento específico & SNS/SGSSS-POS \\
\hline $\begin{array}{l}\text { Enfermedad inflamatoria de los } \\
\text { órganos pélvicos femeninos }\end{array}$ & $614-616$ & N70-N72 & Atención médica & Diagnóstico precoz y tratamiento específico & SNS/SGSSS-POS \\
\hline $\begin{array}{l}\text { Otros trastornos del aparato } \\
\text { genital femenino }\end{array}$ & $617-629$ & N80-N83 & Atención médica & Diagnóstico precoz y tratamiento específico & SNS/SGSSS-POS \\
\hline
\end{tabular}

aumentaron las muertes por homicidios $(\mathrm{PCA}=1,7 \%)$ y las que se hubieran podido evitar mediante medidas de saneamiento ambiental $(\mathrm{PCA}=7,2 \%)$ y medidas mixtas (PCA $=0,9 \%)$.

\section{DISCUSIÓN}

En este trabajo se propone un INIME que busca facilitar la evaluación de las políticas relacionadas con la salud to- mando en cuenta el perfil epidemiológico local y la responsabilidad de diferentes actores sobre los factores determinantes de la salud.

La formulación del INIME a partir de las listas de ME de Holland y de Taucher -que reflejan un consenso de varios años-, el criterio de Uemura — que facilitó la selección de las causas de muerte que han sido aceptadas como indicadores de $\mathrm{ME}$ en los países industrializados y América Latina- y el criterio de Rutstein y colaboradores — que permitió excluir las causas de muerte para las cuales no se identificó por lo menos una medida de intervención que pudiera considerarse efectiva en el momento del análisis, como las anomalías congénitas (código 740-759 de la CIE-9) y los síntomas o síndromes mal definidos (agrupados bajo el código 780-799 de la CIE-9)—, además de criterios de equidad. 
CUADRO 2. Causas evitables de muerte por grupos de enfermedades, recogidas en el inventario de indicadores de mortalidad evitable (INIME)

\begin{tabular}{lll}
\hline Grupo & Causas de muerte según el criterio de evitable & CIE-9a \\
\hline
\end{tabular}

A Defunciones evitables por vacunación o tratamiento preventivo

Todas las zoonosis bacterianas (peste, tularemia, carbunco, brucelosis, muermo, amiloidosis, fiebre por mordedura de rata y otras zoonosis bacterianas); lepra y otras enfermedades por micobacterias difteria; tos ferina; angina estreptocócica y escarlatina; erisipela; tétanos; poliomielitis aguda; viruela; sarampión; rubéola; sífilis y otras enfermedades venéreas; fiebre reumática y corea reumática; pericarditis reumática y otras enfermedades reumáticas del corazón

NC Infección meningocócica; fiebre amarilla; rabia; secuelas de la poliomielitis; hepatitis B; secuelas de la lepra; cardiopatías reumáticas agudas o crónicas

B Defunciones evitables por diagnóstico y tratamiento médico precoz

Tumores malignos de la mama, el útero y la próstata; diabetes mellitus; enfermedad hipertensiva; bronquitis no especificada como aguda o como crónica; bronquitis crónica; enfisema; asma; obstrucción crónica de las vías respiratorias no clasificadas en otra parte; enfermedades de la cavidad bucal, de las glándulas salivales y de los maxilares; enfermedades del esófago distintas a cáncer; úlcera del estómago o del duodeno; gastritis y duodenitis; enfermedades del apéndice; hernias abdominales y obstrucción intestinal; enfermedad diverticular del intestino; trastornos digestivos funcionales no clasificados en otra parte; colelitiasis y otros trastornos de la vesícula biliar; peritonitis; absceso hepático y las secuelas de las hepatopatías tóxicas; insuficiencia renal; infecciones renales; hiperplasia de la próstata

NC Septicemia; otros trastornos del aparato genital femenino; enfermedad hipertensiva y cerebro vascular; enfermedad isquémica del corazón; valvulopatías; insuficiencia cardíaca congestiva; tromboflebitis litiasis renal y ureteral; cistitis; enfermedades de los órganos genitales; trastornos de la mama; enfermedad inflamatoria de los órganos pélvicos femeninos

C Defunciones evitables por medidas de saneamiento ambiental

Cólera; tifoidea y paratifoidea; salmonelosis; shiguelosis; otras intoxicaciones alimentarias bacterianas amebiasis; infecciones intestinales debidas a otros organismos; otras infecciones intestinales mal definidas; hepatitis vírica; los tifus; las ricketsiosis transmitidas por garrapatas; otras ricketsiosis; tripanosomiasis; leishmaniasis; leptospirosis; efectos tardíos de otras enfermedades infecciosas y parasitarias

NC Otras enfermedades intestinales debidas a giardias y otros protozoos

D Defunciones evitables por medidas mixtas

D1 Infecciones de las vías respiratorias (todas las Infecciones respiratorias agudas, neumonías e influenza)

D2 Problemas del embarazo, el parto y el puerperio: mola hidatidiforme; otro producto anormal de la concepción; aborto retenido; embarazo ectópico; aborto espontáneo; aborto inducido legalmente; aborto inducido ilegalmente; aborto no especificado; intento fallido de aborto; complicaciones consecutivas al aborto, a la gestación ectópica y al embarazo molar; hemorragia precoz del embarazo; hemorragia anteparto; desprendimiento prematuro de la placenta y placenta previa; hipertensión que complica el embarazo, el parto y el puerperio; hiperemesis del embarazo; otras complicaciones del embarazo no clasificadas en otra parte; enfermedades infecciosas y parasitarias de la madre clasificables en otra parte cuando complican el embarazo, parto o puerperio; otras afecciones maternas concurrentes clasificables en otra parte cuando complican el embarazo, el parto o el puerperio; parto en condiciones normales; parto obstruido; hemorragia postparto; todas las categorías del grupo de las complicaciones del puerperio (sepsis, hemorragias, embolias y otras)

D3 Enfermedades propias de la primera infancia: feto o neonato afectado por enfermedades de la madre no necesariamente relacionadas con el embarazo actual; feto o neonato afectado por complicaciones maternas del embarazo; feto o neonato afectado por complicaciones de la placenta, del cordón umbilical o de las membranas; feto o neonato afectado por otras complicaciones del trabajo y del parto; crecimiento fetal lento y desnutrición fetal; trastornos relacionados con la duración corta de la gestación y con otra forma de peso bajo al nacer; trastornos del feto o neonato relacionados con el embarazo prolongado y el peso elevado al nacer; traumatismos del nacimiento; hipoxia intrauterina y asfixia al nacer; síndrome de dificultad respiratoria; otras afecciones respiratorias del feto y del recién nacido; infecciones propias del período perinatal; hemorragia fetal y neonatal; enfermedad hemolítica del recién nacido debida a isosensibilización; otras formas de ictericia perinatal; trastornos endocrinos y metabólicos propios del feto y del recién nacido; trastornos hematológicos del feto y del recién nacido; trastornos perinatales del aparato digestivo; afecciones asociadas con la regulación tegumentaria y de la temperatura del feto y del recién nacido; otras afecciones y las mal definidas que se originan en el período perinatal
020-027; 030-031; 032; $033 ; 034 ; 035 ; 037 ; 045$ 050; 055; 056; 090-099; $390 ; 392 ; 393 ; 398$

036; 060; 071; 138; 391; 394-397

$174 ; 180 ; 182 ; 185 ; 250$ $401-405 ; 490 ; 491 ; 492$ $493 ; 496 ; 520-529 ; 530$; $531-533 ; 535 ; 540-543$; 550-553; 560; 562; 564; $574-575 ; 567 ; 572$; 584-586; 590; 600

038; 617-629; 430-438; 410-414; 424; 428; 453; $592 ; 594 ; 595 ; 600-608$; 610-611; 614-616

001; 002; 003; 004; 005 006; 008; 009; 070; 080-081; 082; 083; 086; $085 ; 100 ; 139$

007

$460-466 ; 480-487$

$630 ; 631 ; 632 ; 633 ; 634$ $635 ; 636 ; 637 ; 638 ; 639$; $640 ; 641 ; 642 ; 643 ; 646$; $647 ; 648 ; 650 ; 660 ; 666$; 670-676

$760 ; 761 ; 762 ; 763 ; 764$ $765 ; 766 ; 767 ; 768 ; 769$ 770; 771; 772; 773; 774 $775 ; 776 ; 777 ; 778 ; 779$
A20-A28; A30-A31; A36; A37; A38; A46; A33-A34; A80; B03; B05; B06; A50-A63; 100; I02; 109

A39; A95; A82; B91; B160; B161; B180 B181; B92; I01; I05-I08

C50; C53-C55; C61; E10-E14; I10-I15; J40; J41-J42; J43; J45; J44; J46; J47; K00-K14; K20-K23; K25-K28; K29-K30; K35-K38; K40-K46; K56; K57; K59; K80-K83; K65; K75-K77; N17-N19; N40

A40-A41; N80-N83; I60-I69; I20-I25; I34; I50; I82; N20; N21; N30; N40-N51; N60-N64; N70-N72

$\mathrm{A} 00 ; \mathrm{A} 01 ; \mathrm{A} 02 ; \mathrm{A} 03$ A05; A06; A08; A09; B15; A75; A77-A78; A79; B56-B57; B55; A27; B94; B92

A07

J00-J06; J10-J18; J20-J22

O01; 002; 005; 000; 003; 004; 005; 006; O07; O08; O20; 043; O44; 045; 046; 010; 011; 013; 014; 015; O16; 021; 098; 099; O80; O64-066; 072; 085-092

P00; P01; P02; P03 P05; P07; P08; P10-P15; P20-P21; $\mathrm{P} 22 ; \mathrm{P} 23-\mathrm{P} 28$; P35-P39; P50-P56; P55; P57-P59; P70-P74; P60-P61; P75-P78; P80-P83; P90-P96 


\section{CUADRO 2. (Continuación)}

\begin{tabular}{|c|c|c|c|}
\hline Grupo & Causas de muerte según el criterio de evitable & CIE-9a & $\mathrm{CIE}-10^{\mathrm{b}}$ \\
\hline NC & Delírium trémens; síndrome de abstinencia de la droga & $291 ; 292$ & \\
\hline D4 & $\begin{array}{l}\text { Causas externas: todos los traumatismos y envenenamientos; síndrome de dependencia del alcohol; } \\
\text { dependencia y abuso de drogas }\end{array}$ & 800-999; 303; 304; 305 & V00-Y98; F10; F11-F19 \\
\hline D5 & $\begin{array}{l}\text { Tuberculosis: tuberculosis primaria, tuberculosis pulmonar, otras tuberculosis del aparato respiratorio, } \\
\text { tuberculosis del sistema nervioso central, tuberculosis gastrointestinal, tuberculosis genitourinaria, } \\
\text { tuberculosis miliar }\end{array}$ & $\begin{array}{l}010 ; 011 ; 012 ; 013 ; 014 ; \\
016 ; 018\end{array}$ & $\begin{array}{l}\text { A15-A16; A15-A16; } \\
\text { A15-A16; A17; A18; } \\
\text { A18; A19 }\end{array}$ \\
\hline NC & Tuberculosis de los huesos y las articulaciones; tuberculosis de otros órganos; secuelas de la tuberculosis & $015 ; 017 ; 137$ & A18; A18; B90 \\
\hline D6 & $\begin{array}{l}\text { Problemas carenciales: kwashiorkor; marasmo; otra desnutrición proteico-calórica grave; otras formas } \\
\text { de desnutrición proteico-calóricas y las no especificadas; otras anemias por deficiencia; otras anemias } \\
\text { y las no especificadas; otras enfermedades de la sangre y de los órganos hematopoyéticos }\end{array}$ & $\begin{array}{l}260 ; 261 ; 262 ; 263 ; 281 ; \\
285 ; 289.4\end{array}$ & $\begin{array}{l}\text { E40; E41-E42; E43; } \\
\text { E44-E46; D51-D53; } \\
\text { D461; D463; D731 }\end{array}$ \\
\hline NC & Anemia por deficiencia de hierro & 280 & D50 \\
\hline D7 & Problemas hepáticos: cirrosis y otras enfermedades crónicas del hígado & 571 & K70-K73 \\
\hline D8 & Enfermedades relacionadas con el medio ambiente & & \\
\hline NC & Dengue; paludismo; leishmaniasis; todas las helmintiasis; toxoplasmosis; efectos tardíos del tracoma & $\begin{array}{l}061 ; 0654 ; 084 ; 085 \\
120-129 ; 130 ; 139\end{array}$ & $\begin{array}{l}\text { A90-A91; B50-B54; } \\
\text { B55; B65-B83; B58; B94 }\end{array}$ \\
\hline D9 & VIH-sida (desde 1988) & $279.5 ; 279.6 ; 795.8$ & B20-B24 \\
\hline D10 & Tumores relacionados con el tabaquismo & & \\
\hline NC & Tumores de laringe; tumores de tráquea; tumores de bronquios y pulmón & $161 ; 162.0 ; 162.1-162.9$ & C32; C33; C34 \\
\hline \multirow[t]{2}{*}{$E^{d}$} & Defunciones difícilmente evitables en la actualidad & & \\
\hline & $\begin{array}{l}\text { Tumores malignos (excepto los del grupo B); tumores malignos de la cavidad bucal y de la faringe; } \\
\text { tumores malignos de otros órganos digestivos y del peritoneo; tumores malignos de los órganos } \\
\text { respiratorios e intratorácicos; tumores malignos de los huesos; del tejido conectivo y de la piel (excepto } \\
\text { el de mama); tumores malignos de los órganos genitourinarios (excepto los de útero y próstata); } \\
\text { tumores malignos de otros sitios y los no especificados; tumores malignos del tejido linfático y de los } \\
\text { órganos hematopoyéticos; enfermedad de Parkinson; esclerosis múltiple; epilepsia; enfermedad } \\
\text { arterioesclerótica y degenerativa del corazón y otras enfermedades del corazón; enfermedades cerebro } \\
\text { vasculares; aterosclerosis; malformaciones congénitas del sistema nervioso o cardiovascular }\end{array}$ & $\begin{array}{l}140-149 ; 150-159 ; \\
160-165 ; 170-173 ; 175 \\
181 ; 183 ; 184 ; 186-189 \\
190-199 ; 200-208 ; 332 \\
340 ; 345 ; 410-416 ; \\
420-429 ; 430-438 ; 440 \\
740-742 ; 745-747\end{array}$ & $\begin{array}{l}\text { C00-C14; C15-C26; } \\
\text { C48-C49; C30-C39; } \\
\text { C40-41; C43-C44; } \\
\text { C45-C47; C51-C52; } \\
\text { C56-C60; C62-C75; } \\
\text { C76-C80; C97; } \\
\text { C81-C96; G20; G35; } \\
\text { G40; I20-I25; I30-I52; } \\
\text { I60-I69; I70; Q00-Q07; } \\
\text { Q20-Q28 }\end{array}$ \\
\hline \multirow[t]{2}{*}{$l^{d}$} & Defunciones por causas mal definidas & & \\
\hline & Otros síntomas o síndromes y los mal definidos & $780-799$ & R00-R99 \\
\hline \multirow[t]{2}{*}{$J^{d}$} & Otras causas & & \\
\hline & Todas las demás enfermedades y códigos no incluidos en los grupos anteriores & Resto de las categorías & Resto de las categorías \\
\hline
\end{tabular}

${ }^{a}$ CIE-9: Clasificación Internacional de Enfermedades, 9. ${ }^{a}$ revisión (27).

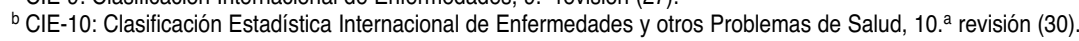

${ }^{c}$ NC: Nuevas categorías: causas de muerte evitable no incluidas en las listas de Holland y de Taucher.

d Los grupos E, I y J son condiciones de muerte consideradas no evitables, pero se incluyen en el cuadro para que la clasificación sea exhaustiva.

Para la construcción del INIME se enfrentaron varios problemas conceptuales y metodológicos que restringen su alcance y que podrían agruparse en cuatro categorías: a) las diferencias en los enfoques sobre la $\mathrm{ME}$; b) la vinculación del carácter evitable de una muerte a una tecnología específica potencialmente efectiva; c) la naturaleza de los datos uti- lizados para seleccionar los indicadores; y d) la aplicabilidad de la nueva lista.

\section{Diferencia de enfoques sobre la ME}

Para diseñar el INIME fue necesario adoptar una noción de ME y definir el alcance teórico de los indicadores. La noción de ME adoptada fue la acuñada originalmente por Rutstein y colaboradores (14) en la que se considera como parte de la AM las actividades de prevención primaria y el tratamiento a cargo de diferentes agentes estatales y privados. Este concepto se enfoca predominantemente en las intervenciones de los servicios de salud, a diferencia de la mayoría de los estudios de $\mathrm{ME}$, que se 
CUADRO 3. Comparación de las listas de mortalidad evitable (ME) de Holland y de Taucher y el inventario de indicadores de mortalidad evitable (INIME) aplicados a las defunciones registradas en Colombia entre 1993 y 1996

\begin{tabular}{|c|c|c|c|c|c|c|c|}
\hline \multirow[b]{2}{*}{ Categoría a comparar } & \multirow[b]{2}{*}{ No. } & \multirow[b]{2}{*}{$\%$} & \multicolumn{3}{|c|}{$\begin{array}{c}\text { Muertes clasificadas } \\
\text { como evitables, \% }\end{array}$} & \multicolumn{2}{|c|}{$\begin{array}{c}\text { Diferencia porcentual en } \\
\text { la ME captada por el } \\
\text { INIME con respecto a }\end{array}$} \\
\hline & & & $\begin{array}{l}\text { Según la } \\
\text { lista de } \\
\text { Holland }^{\mathrm{a}}\end{array}$ & $\begin{array}{l}\text { Según la } \\
\text { lista de } \\
\text { Taucher }^{\text {b }}\end{array}$ & $\begin{array}{l}\text { Según el } \\
\text { INIME }\end{array}$ & $\begin{array}{l}\text { Lista de } \\
\text { Holland }\end{array}$ & $\begin{array}{l}\text { Lista de } \\
\text { Taucher }\end{array}$ \\
\hline Total de defunciones & 680617 & 100,0 & 18,2 & 51,3 & 76,7 & 58,5 & 25,4 \\
\hline \multicolumn{8}{|l|}{ Año } \\
\hline 1993 & 168647 & 24,8 & 18,2 & 51,7 & 76,2 & 58,0 & 24,5 \\
\hline 1994 & 168568 & 24,8 & 18,4 & 51,1 & 76,3 & 57,9 & 25,1 \\
\hline 1995 & 169896 & 25,0 & 18,3 & 51,1 & 76,7 & 58,4 & 25,7 \\
\hline 1996 & 173506 & 25,5 & 17,8 & 51,3 & 77,5 & 59,7 & 26,3 \\
\hline \multicolumn{8}{|l|}{ Sexo } \\
\hline Varones & 412462 & 60,6 & 17,3 & 57,8 & 80,2 & 62,9 & 22,4 \\
\hline Mujeres & 268155 & 39,4 & 19,6 & 41,3 & 71,3 & 51,7 & 29,9 \\
\hline \multicolumn{8}{|l|}{ Edad (años) } \\
\hline De 0 a 4 & 57643 & 8,5 & 47,1 & 69,0 & 70,3 & 23,2 & 1,3 \\
\hline De 5 a 9 & 6035 & 0,9 & 40,2 & 60,4 & 63,9 & 23,7 & 3,5 \\
\hline De 10 a 14 & 6795 & 1,0 & 30,8 & 65,5 & 69,4 & 38,6 & 3,9 \\
\hline De 15 a 19 & 24951 & 3,7 & 12,4 & 86,8 & 89,2 & 76,8 & 2,4 \\
\hline De 20 a 24 & 35242 & 5,2 & 12,1 & 88,4 & 91,7 & 79,6 & 3,2 \\
\hline De 25 a 29 & 33423 & 4,9 & 14,7 & 84,4 & 90,0 & 75,3 & 5,6 \\
\hline De 30 a 34 & 30289 & 4,5 & 16,9 & 78,5 & 87,3 & 70,4 & 8,8 \\
\hline De 35 a 39 & 28167 & 4,1 & 24,3 & 72,3 & 84,2 & 60,0 & 11,9 \\
\hline De 40 a 44 & 24420 & 3,6 & 30,4 & 63,0 & 80,1 & 49,7 & 17,1 \\
\hline De 45 a 49 & 24182 & 3,6 & 37,3 & 53,8 & 77,0 & 39,7 & 23,2 \\
\hline De 50 a 54 & 27477 & 4,0 & 42,7 & 44,9 & 73,8 & 31,1 & 28,9 \\
\hline De 55 a 59 & 33181 & 4,9 & 44,5 & 39,1 & 72,1 & 27,6 & 33,1 \\
\hline De 60 a 64 & 44395 & 6,5 & 45,5 & 35,8 & 71,6 & 26,1 & 35,9 \\
\hline De 65 a 69 & 52732 & 7,8 & 3,1 & 34,5 & 72,2 & 69,1 & 37,7 \\
\hline De 70 a 74 & 58809 & 8,6 & 2,4 & 34,0 & 72,5 & 70,1 & 38,6 \\
\hline De 75 a 79 & 60740 & 8,9 & 1,0 & 34,0 & 73,6 & 72,6 & 39,6 \\
\hline De 80 a 84 & 55187 & 8,1 & 0,8 & 34,0 & 74,2 & 73,4 & 40,2 \\
\hline$\geq 85$ & 62441 & 9,2 & 0,4 & 34,0 & 74,0 & 73,6 & 40,0 \\
\hline \multicolumn{8}{|l|}{ Grupo de causas } \\
\hline I. Infecciosas y parasitarias & 26539 & 3,9 & 33,6 & 73,3 & 77,8 & 44,2 & 4,5 \\
\hline II. Tumores & 95265 & 14,0 & 17,0 & 19,1 & 30,9 & 13,9 & 11,9 \\
\hline III. Endocrinas y metabólicas & 22276 & 3,3 & 0,1 & 97,1 & 97,1 & 97,1 & 0,0 \\
\hline IV. Sangre y hematopoyéticas & 4689 & 0,7 & 0,8 & 31,6 & 31,1 & 31,3 & 0,5 \\
\hline V. Trastornos mentales & 316 & 0,0 & 0,6 & 43,7 & 44,6 & 44,0 & 0,9 \\
\hline \multicolumn{8}{|l|}{ VI. Sistema nervioso central y órganos } \\
\hline de los sentidos & 7478 & 1,1 & 0,5 & 0,0 & 0,0 & $-0,5$ & 0,0 \\
\hline VII. Circulatorias & 201318 & 29,5 & 21,4 & 10,6 & 87,6 & 66,3 & 77,0 \\
\hline VIII. Respiratorias & 56712 & 8,3 & 7,8 & 84,5 & 84,5 & 76,7 & 0,0 \\
\hline IX. Digestivas & 32063 & 4,7 & 24,4 & 51,0 & 52,2 & 27,7 & 1,1 \\
\hline X. Genitourinarias & 8601 & 1,3 & 0,2 & 100,0 & 100,0 & 99,8 & 0,0 \\
\hline XI. Embarazo, parto y puerperio & 1884 & 0,3 & 100,0 & 100,0 & 100,0 & 0,0 & 0,0 \\
\hline XII. Piel y tejido celular subcutáneo & 579 & 0,1 & 0,3 & 0,0 & 0,0 & $-0,3$ & 0,0 \\
\hline XIII. Osteomusculares y tejido conjuntivo & 48 & 0,0 & 0,0 & 0,0 & 0,0 & 0,0 & 0,0 \\
\hline XIV. Anomalías congénitas & 6583 & 1,0 & 35,7 & 0,0 & 0,0 & $-35,7$ & 0,0 \\
\hline XV. Perinatales & 20287 & 3,0 & 67,6 & 100,0 & 100,0 & 32,4 & 0,0 \\
\hline XVI. Mal definidas & 24212 & 3,6 & 1,1 & 0,0 & 0,0 & $-1,1$ & 0,0 \\
\hline XVII. Externas & 171745 & 25,2 & 14,6 & 100,0 & 100,0 & 85,4 & 0,0 \\
\hline
\end{tabular}

a Véanse las referencias 9 y 10.

${ }^{b}$ Véanse las referencias 12, 23 y 24.

han basado en la formulación y la medición de indicadores de fallas en la AM, lo que refleja en ocasiones la influencia de enfoques deterministas y unicausales que centran el carácter evitable de la mortalidad en el acto médico. Estos enfoques han facilitado la detección de fallas en la AM, pero pueden tener una utilidad limitada para evaluar las políti- cas públicas que actúan sobre los factores determinantes de la salud. Por esta razón, en este estudio se optó por una aproximación multicausal de la $\mathrm{ME}$, en el que a la vez que se reconoce que la AM puede evitar un buen número de muertes y toma en cuenta otros factores biológicos, sociales y ambientales que influyen en el resultado.
A partir de los argumentos anteriores, en este estudio se adoptó una definición de ME con un criterio amplio y se seleccionaron varias causas de muerte que pudieran servir como posibles indicadores de fallas en el control de la mortalidad, tanto dentro del sistema de la AM como en otros sectores. Así, la expresión "todo lo que debería hacerse" se aplicó también 
a las intervenciones realizadas fuera del sector salud que pudieran contribuir a reducir los riesgos de enfermar y morir, o mejorar el acceso a los servicios. Este criterio permitió clasificar como evitables varias causas de muerte no contempladas en las listas de Holland y de Taucher, entre ellas las muertes por formas extrapulmonares de tuberculosis, fiebre amarilla, malaria y anemia por déficit de hierro, que en Colombia constituyen problemas importantes de salud pública.

A diferencia de la lista de ME de Holland, que no reconoce muertes evitables en personas de 65 años o más, el INIME no consideró la edad como criterio restrictivo para clasificar una muerte como evitable o no. Aunque se reconoce que algunas intervenciones pueden ser menos eficaces en las personas de edad avanzada, la decisión de no restringir la condición de evitable según la edad se tomó por criterios éticos de defensa de los derechos de los adultos mayores a las intervenciones médicas y sanitarias, y evitar planteamientos que promuevan la aceptación de la muerte en esas personas como una fatalidad cuyo riesgo no amerita intervención. El presente estudio muestra que si se acepta de manera rígida el límite de 65 años para considerar una muerte evitable habría que considerar absolutamente inevitable entre $7,8 \%$ y $9,1 \%$ de las defunciones registradas en mayores de 65 años en Colombia por causas consideradas evitables mediante la AM en otros grupos. Sin embargo, las grandes diferencias entre las proporciones de muertes evitables obtenidas en este grupo de edad según las dos listas de ME y el INIME se concentran en las causas de muerte relacionadas con el control de los factores determinantes sociales y ambientales, ya que entre $69,1 \%$ y $73,6 \%$ de las muertes registradas en mayores de 65 años podrían considerarse evitables mediante intervenciones de sectores diferentes al de salud.

La menor respuesta de los adultos mayores al tratamiento médico es un problema real y está relacionado con la efectividad de la AM; pero esto no implica que las muertes por desnutrición, homicidio, accidentes, carencias socioeconómicas o exposición a un ambiente malsano en mayores de 65 años constituyan, por la sola razón de la edad, hechos inevitables. La inequidad que pudiera derivarse de reconocer el carácter evitable en unos grupos y no evitable en otros constituye un aspecto especialmente controversial que amerita mayor discusión.

\section{Vínculo entre la ME y las tecnologías potencialmente efectivas}

No resulta fácil identificar al menos una tecnología potencialmente efectiva que pueda modificar el riesgo de muerte para cada causa, como proponen Rutstein y colaboradores y Holland. Esto se debe a que los determinantes sociales, económicos y ecológicos de la mortalidad operan mediante procesos biológicos básicos y variables mediadoras, cuya interacción influye en la efectividad de las intervenciones dirigidas a reducir los riesgos de enfermar y morir. En algunos casos, la relación entre la aplicación de una tecnología y la subsecuente reducción de la mortalidad es muy clara, como lo ilustra la brusca disminución de la mortalidad por difteria observada en el mundo después de la introducción de la vacuna. En otros casos, la relación entre la intervención y el efecto es confusa (31, 32), pues la efectividad, más que una propiedad mecánica de las intervenciones, depende de quién realiza las acciones y de cómo, cuándo y en qué condiciones se realizan. La mayoría de las muertes maternas, por ejemplo, se podrían evitar con medidas de AM; sin embargo, las condiciones geográficas, económicas, administrativas y culturales pueden impedir a muchas madres acceder oportunamente a esos servicios. En este caso, el indicador de ME puede reflejar fallas en las políticas sociales, más que deficiencias del acto médico.

En condiciones reales, determinar el carácter evitable de una muerte es un procedimiento especialmente complejo que no puede atribuirse mecánicamente a una sola intervención. Para facilitar la elaboración de las listas de ME, los expertos suelen valorar el carácter evitable de una causa de muerte con criterios operativos que privilegian el tipo de intervención específica que en su opinión es más efectiva. A esto se suma que la mayoría de esas listas parten de que las causas consideradas evitables lo son en todos los casos. Sin embargo, clasificar una causa de muerte como evitable no significa que siempre lo sea. Estrictamente hablando, el grado en que una intervención puede modificar los riesgos de enfermar o morir por una causa dada solo puede establecerse mediante estudios apropiados de efectividad que valoren el peso relativo de los diferentes factores involucrados en el resultado. Aunque los criterios de Tobias y Jackson
$(11,33)$ abren un camino en este sentido, las evidencias sobre la efectividad poblacional son aún escasas e insuficientes para fundamentar los indicadores de ME. En ausencia de otra evidencia, las listas de ME suelen resolver este problema mediante el consenso de expertos, método que puede resultar útil para fundamentar las decisiones (34), pero que no está exento de sesgos y limitaciones.

Al calificar el carácter evitable o no de las defunciones registradas, en este estudio se encontró que un buen número de las causas de muerte podía responder en grado variable a diferentes acciones, algunas de las cuales eran predominantemente médicas, mientras otras se relacionaban con políticas e intervenciones relacionadas con los factores determinantes sociales y ambientales. Por su finalidad pragmática, en el estudio se admitieron como proveedores de tecnologías potencialmente efectivas las agencias estatales, privadas o comunitarias que actuaban de manera explícita por lo menos sobre uno de los factores determinantes del proceso que conduce a la muerte. Aunque para una misma causa evitable se identificaron con frecuencia varias intervenciones diferentes potencialmente efectivas, el INIME se conformó fundamentalmente a partir de los grupos de intervenciones propuestos por Taucher, ya que promueven un enfoque más integral y pragmático de la ME y responden a las causas predominantes en la estructura de mortalidad de Colombia en el período analizado.

Llama la atención que para cada una de las causas consideradas evitables por Holland, por Taucher y por el INIME se identificó en Colombia por lo menos un proveedor (con frecuencia más de uno) de intervenciones relacionadas con su control. La proporción de muertes evitables, en ocasiones elevada, encontrada en una población en la que se identifican proveedores de servicios potencialmente efectivos puede indicar, sin embargo, que esta condición no garantiza por sí misma el impacto de sus acciones, el cual puede estar mediado por el acceso y la calidad, lo que puede demandar una mayor atención al analizar la ME.

\section{Naturaleza de los datos utilizados para seleccionar los indicadores}

Los indicadores del INIME se basaron en la CIE-9, que era la clasificación utilizada por el sistema de estadísticas vitales 
en Colombia al momento del estudio. Al clasificar las defunciones, la CIE presume que la muerte se debe a "todas aquellas enfermedades, estados morbosos o lesiones que produjeron la muerte o contribuyeron a ella, y las circunstancias del accidente o de la violencia que produjo dichas lesiones" (27). Esencialmente, sus códigos reflejan la perspectiva médica y, con excepción de los capítulos de causas externas y otras causas, brindan poca información acerca de los demás factores que determinaron la defunción. El diagnóstico médico que conduce a la causa básica de la defunción es sólo uno de los eslabones de una compleja cadena de eventos de diferente naturaleza que se estructuran y afectan mutuamente hasta producir la muerte, cuya dinámica escapa con frecuencia a la CIE. La causalidad múltiple, los riesgos competitivos y, especialmente, la influencia de factores determinantes no biológicos, son elementos que limitan el alcance de los indicadores de ME basados en la CIE.

\section{Aplicabilidad de la nueva lista}

La validez externa del INIME no se puede establecer en este artículo. A pesar de la importancia de las listas de ME y su amplia utilización en varios países, no existe un estándar que permita establecer la validez de los criterios de sus indicadores. En tal sentido, el INIME puede presentar ventajas y limitaciones similares a las que presentan las listas originales de Holland y de Taucher. A este respecto, ni la intencionalidad del presente estudio ni el método utilizado para seleccionar los indicadores pretenden asegurar que el INIME tenga validez universal.

Este estudio supone, en cambio, que los indicadores incluidos en el INIME constituyen un recurso útil para facilitar el análisis y que su significado se revela desde los contextos y la historia del grupo analizado. Estos conceptos, que se han desarrollado en otros campos de la salud pública por la epidemiología multinivel (35) y los enfoques epidemiológicos históricosociales (36), tienen implicaciones importantes al momento de aplicar el INIME e interpretar estos resultados. En primer lugar, indican que el analista no debe atribuir mecánicamente a una sola intervención las variaciones observadas en la mortalidad; en segundo lugar, implican que cada uno de los indicadores incluidos en las listas de ME puede tener su propia validez y utilidad en un contexto, sin ser igualmente válido y útil en otros. La tarea del analista será identificar y seleccionar los indicadores del INIME que mejor reflejen la situación del caso en estudio.

La validez de las mediciones de la ME se relaciona también con su capacidad para reflejar el contexto epidemiológico y los determinantes de la mortalidad objetos de interés para el analista. En ese sentido, la interpretación de las mediciones de ME debe hacerse desde los contextos específicos del caso de estudio. Si los perfiles epidemiológicos de dos poblaciones estudiadas difieren en cuanto a la estructura y los niveles de la mortalidad - por ejemplo, por las enfermedades susceptibles de AM, los accidentes, los homicidios o las enfermedades entéricas- no se debe esperar que las proporciones de muertes evitables sean iguales en ambas.

La comparación de las proporciones de muerte evitables obtenidas según las dos listas de ME y el INIME puso de manifiesto marcadas diferencias que reflejan sus diferentes enfoques al concebir la ME y que tienen implicaciones que los tomadores de decisiones deben tener en cuenta. Debido a que en el INIME se incluyeron varias causas de muerte evitables por acciones diferentes de la AM, se clasifica como muertes evitables una mayor proporción de defunciones en la población analizada que según las otras dos listas.

Las mediciones de la ME reflejan la prevalencia y la incidencia de las causas de muerte en cada región; un efecto que ya ha sido descrito (21). La alta frecuencia de homicidios, defunciones en mayores de 65 años, trastornos cardiovasculares y enfermedades infecciosas y parasitarias en la población analizada puede explicar en parte la alta proporción de muertes evitables obtenida con el INIME y justifica la pertinencia de contar con una lista de ME que incluya indicadores para estas causas. Las diferencias entre el INIME y la clasificación de Taucher están relacionadas principalmente con problemas del aparato circulatorio y tumores, que no son reconocidas como ME por esa lista. Estas diferencias fueron más marcadas en las mujeres de 45 a 65 años debido fundamentalmente a las muertes por trastornos cardiovasculares. Las diferencias entre INIME y la clasificación de Holland se explican principalmente porque esta lista se centra en las causas controlables por AM y no en las evitables mediante acciones de otros sectores.
Estos resultados indican que la aplicación en Colombia de las listas de Holland y de Taucher para evaluar el desempeño de las políticas sanitarias sin realizar los ajustes necesarios a esos instrumentos llevaría a pasar por alto eventos importantes de salud pública que persisten en la sociedad colombiana, a pesar de haber sido controlados en otros países. Ejemplos de tales eventos son el elevado número de homicidios y muertes por malaria y afecciones cardiovasculares en mujeres, y en adultos mayores. Durante el período analizado, los homicidios, las enfermedades infecciosas y parasitarias y las muertes en mayores de 65 años representaron más de la mitad de las muertes registradas en Colombia, mientras aún persisten problemas de acceso a los servicios de AM. Esto puede explicar, además, la diferencia entre los presentes resultados y las mediciones de ME realizadas en Europa, ya que las listas de ME aplicadas allá no suelen tener en cuenta indicadores para estas causas.

El carácter evitable de estas defunciones se revela desde el planteamiento de Uemura, es decir, si estos eventos han sido controlados en gran medida en Europa y los países industrializados, ¿por qué no se pueden considerar evitables en Colombia? La diferencia entre la proporción de muertes evitables por acciones de AM según los criterios de Holland $(18,2 \%)$ y la proporción de muertes evitables por todas las causas potencialmente evitables según el INIME $(76,7 \%)$ refleja no solo el peso que tiene en $\mathrm{Co}-$ lombia la mortalidad por causas no médicas, sino también la capacidad de cada lista de facilitar la identificación de estas muertes como evitables. En todo caso, serán los objetivos del analista los que determinen cuáles indicadores son más útiles para sus fines.

A este respecto se debe resaltar que el INIME no debe emplearse como un indicador de ME, sino como un inventario de indicadores para que el analista seleccione los que pudieran ser más apropiados en su región o país. En principio, el uso de algunos de los indicadores específicos de ME incluidos en el inventario y seleccionados con diferentes fines podría presentar ventajas frente a indicadores más generales, como la tasa de mortalidad general ajustada por la edad o a la esperanza de vida. Es necesario avanzar en el análisis de la ME como una herramienta especialmente importante para evaluar el desempeño de las intervencio- 
nes dirigidas a reducir el riesgo de morir. Sin embargo, estas deben realizarse con un criterio amplio de manera que no solamente reflejen el papel de los determinantes biológicos, sociales y ambientales, sino también que respondan al contexto local en que se aplican las tecnologías.

En conclusión, las causas de ME podrían reflejar fallas no solo en la AM, sino también en el control de otros deter- minantes de la muerte. Mientras se dispone de otros medios para evaluar la efectividad de intervenciones y políticas en salud, los inventarios de ME pueden constituir un recurso valioso. No obstante, se debe tener en cuenta que los resultados de las mediciones pueden variar ampliamente de acuerdo con la intencionalidad del analista, el enfoque epistemológico que soporta sus méto- dos, los criterios adoptados para definir los indicadores, el desempeño de las tecnologías en diversos contextos y las características de los datos disponibles. Los indicadores incluidos en el INIME pueden facilitar el análisis y la evaluación de las políticas sanitarias por causas de muerte que son importantes en la región, pero que no figuran en las listas originales de ME de Holland y de Taucher.

\section{REFERENCIAS}

1. World Health Organization. The world health report 2000. Geneva: WHO; 2001.

2. Petticrew M, Whitehead M, Macintyre S, Graham H, Egan M. Evidence for public health policy on inequalities: 1 : the reality according to policymakers. J Epidemiol Community Health. 2004;58(10):811-6.

3. Macintyre S, Chalmers I, Horton R, Smith RA. Using evidence to inform health policy: case study. Br Med J. 2001;322(222):225.

4. Tang KK, Chin TC, Rao DSP. Avoidable mortality risks and measurement of wellbeing and inequality. J Health Econ. 2008;27(3):624-41.

5. Weisz D, Gusmano MK, Rodwin VG, Neuberg LG. Population health and the health system: a comparative analysis of avoidable mortality in three nations and their world cities. Eur J Public Health. 2008;18(2):166-72.

6. Nolte E, McKee M. Does health care save lives? Avoidable mortality revisited. London: Nuffield Trust; 2004.

7. Nolte E, McKee M. Measuring the health of the nations: how much is attributable to health care? An analysis of mortality amenable to medical care. Br Med J. 2003; 327:1129.

8. Nolte E, McKee C. Measuring the health of nations: updating an earlier analysis. Health Aff (Millwood). 2008;27(1):58-71.

9. Gispert R, Barés MA, Freitas Ramírez A, Torné Farré M, Puigdefàbregas A, Alberquilla A, et al. Medida del resultado de las intervenciones sanitarias en España: una aproximación mediante el análisis temporal y espacial de la mortalidad evitable entre 1986-2001. Rev Esp Salud Publica. 2006;80(2):139-55.

10. Holland WW, EC Working Group on Health Services and "Avoidable Deaths". European Community atlas of avoidable death. 2nd ed. Oxford: Oxford University Press; 1991. (Oxford Medical Publications, Commission of the European Communities Health Services Research Series No. 6, 9.)

11. Tobias M, Jackson G. Avoidable mortality in New Zealand, 1981-1997. Aust N Z J Public Health. 2001;25(1):12-20.

12. Taucher E. Chile: mortalidad desde 1955 a 1975. Tendencias y causas. Santiago: Celade; 1978. (Reporte No.162, Serie A).

13. Malta DC, Duarte EC. Causas de mortes evitáveis por ações efetivas dos serviços de saúde: uma revisão da literatura. Cien Saude Colet. 2007;12(3):765-76.

14. Rutstein DD, Berenberg W, Chalmers TC, Child CG, Fishman AP, Perrin EB. Measuring the quality of medical care: a clinical method. N Eng J Med. 1976;294:582-8.

15. Holland WW, Paul EA, Lakhani A. Avoidable deaths. Lancet. 1988;2(8603):169.

16. Holland WW. Problems and opportunities for health services research in Europe. Public Health Rev. 1993;21(3-4):271-82.

17. Uemura K, Pisa Z. Recent trends in cardiovascular diseases mortality in 27 industrialized countries. World Health Stat Q. 1985; 38(2):142-62.

18. Treurniet HF, Looman CW, van der Maas PJ, Mackenbach JP. Variations in "avoidable" mortality: a reflection of variations in incidence? Int J Epidemiol. 1999;28(2):225-32.

19. Meriel K, Kelvyn J. Impact of definition on the study of avoidable mortality: geographical trends in British deaths 1981-1998 using Charlton and Holland's definitions. Soc Sci Med. 2006;62(6):1443-56.

20. Kunst AE, Looman CW, Mackenbach JP. Medical care and regional mortality differences within the countries of the European Community. Eur J Popul. 1988;4(3):223-45.

21. Holland WW. The contribution of incidence variations to "avoidable mortality". Int J Epidemiol. 1999;28(5):992.

22. Mackenbach JP, Bouvier-Colle MH, Jougla E. "Avoidable" mortality and health services: a review of aggregate data studies. J Epidemiol Community Health. 1990;44(2):106-11.

23. Rodríguez L. México: contribución de las causas de muerte al cambio en la esperanza de vida, 1970-1982. Santiago: Centro Latinoamericano de Demografía; 1989. (Serie B569.)

24. García Molina CA. Causas de muerte evitables y su contribución al incremento en la esperanza de vida. Una interpretación sociodemográfica. El caso de la frontera norte, 1980-1990. Estud Demogr Urbanos. 1995; 10(1):133-66.

25. Sofaer S. Performance indicators: a commentary from the perspective of an expanded view of health. Washington, D.C.: Center for the Advancement of Health; 1995.

26. Durch J, Bailey L, Sotto M. Improving health in the community: a role for performance monitoring. Washington, D.C.: National Academy Press; 1997.

27. Organización Mundial de la Salud. Clasificación internacional de enfermedades. 9. ${ }^{\text {a }}$ revisión. Ginebra: OMS; 1978.

28. Gómez-Arias RD. La mortalidad evitable como indicador de desempeño de la política sanitaria. Colombia, 1985-2001. Medellín: Universidad de Antioquia; 2008.

29. Uemura K, Pisa Z. Trends of mortality from ischaemic disease and other cardiovascular diseases in 27 countries from 1968 to 1977. World Health Stat Q. 1982;35(1):11-47.

30. Organización Panamericana de la Salud. Clasificación estadística internacional de enfermedades y problemas relacionados con la salud. 10. ${ }^{\text {a }}$ revisión. Washington, D.C.: OPS; 1995.

31. Mackenbach JP, Looman CW, Kunst AE. Changes in mortality of diseases which have become (partially) preventable or treatable by medical care, 1950-1984. Ned Tijdschr Geneeskd. 1988;132(36):1665-9.

32. Mackenbach JP. Regional differences in the frequency of various common surgical procedures, 1985. Ned Tijdschr Geneeskd. 1990; 134(19):953-7.

33. Ward J, Girgis S, Adily A. Attributable fractions for premature mortality avoidable through public health action: updated estimates by Australian public health physicians. Aust N Z J Public Health. 2006;30(5):407-12.

34. Gispert R, Barés MA, Puigdefàbregas A, Grupo para el Consenso en la Mortalidad Evitable. La mortalidad evitable: lista de consenso para la actualización del indicador en España. Gac Sanit. 2008;20(3):184-93.

35. Susser M. Choosing a future for epidemiology: II. From black box to Chinese boxes and ecoepidemiology. Am J Public Health. 1996; 86:674-7.

36. Honjo K. Social epidemiology: definition, history, and research examples. Environ Health Prev Med. 2004;9:193-9.

Manuscrito recibido el 22 de julio de 2008. Aceptado para publicación, tras revisión, el 21 de febrero de 2009. 
ABSTRACT Objectives. To develop a list of indicators of avoidable mortality (LIAM) in order to analyze failed efforts to control the mortality risks prevalent in Colombia, and to compare its results to those of two widely-used approaches.

Design and comparative analysis of an inventory of avoidable mortality indicators specific to health conditions in Colombia

Methods. The official mortality records of Colombia for 1985-2001 were reviewed; the basic causes of death were classified according to the ICD-9. Indicators of avoidable mortality (AM) were selected using an algorithm that combined the lists of Holland and Taucher, the definition of Rutstein and colleagues, and the principle of Uemura. The proportions of avoidable deaths that resulted from applying the LIAM and the two AM lists were compared to a database containing Colombia's official death records from 1993-1996.

Results. Of the 680617 deaths registered during the study period, $18.2 \%$ were classified as avoidable according to Holland's list, and 51.3\%, by Taucher's list. According to the LIAM, avoidable mortality rose to $76.7 \%$. This pattern remained relatively stable in 1993-1996. The differences observed between the proportions of avoidable deaths according to the LIAM and that of the two other lists were related to the local epidemiological profile and the conceptual approach of each list.

Conclusions. The differences between the LIAM and the AM lists of Taucher and of Holland attest to the consequences of using one or another classification in the Colombian context. The LIAM can be a valuable resource for undergirding and evaluating health policies, but must be adjusted to the specific situation in which it is applied.

Key words Mortality; health evaluation; health policy; social inequity; Colombia. 